\title{
Temperature of ion cyclotron heated impurity ions in fusion plasmas
}

\author{
D. Pilipenko \\ Université Libre de Bruxelles, Association EURATOM-Etat Belge, Campus Plain CP231, \\ 1050 Brussels, Belgium
}

\section{Z. Tokar}

Institut für Plasmaphysik, Forschungszentrum Jülich GmbH, Association FZJ-Euratom, 52425 Jülich, Germany

I. Pavlenko a) and B. Weyssow

Université Libre de Bruxelles, Association EURATOM-Etat Belge, Campus Plain CP231, 1050 Brussels, Belgium

(Received 14 April 2005; accepted 26 May 2005; published online 13 July 2005)

\begin{abstract}
The temperature of impurity ions in fusion plasmas is normally assumed to be the same as that of background particles. This assumption can be, however, violated for selective impurity species intensively heated by radio frequency waves or in reactor plasmas of low collisionality. An approach to compute the impurity temperature under such conditions is elaborated. It is based on the construction of a hierarchy of approximate solutions to the impurity heat balance equation and takes into account that the density and, thus, the heat conductivity of heated ion species can change by many orders of magnitude with the position in the plasma. Coupled with the wave propagation code, the particle and heat balances for impurity and main plasma species provide a self-consistent approach to model the ion cyclotron resonance heating scenario. (C) 2005 American Institute of Physics. [DOI: 10.1063/1.1978447]
\end{abstract}

\section{INTRODUCTION}

Experiments on several tokamaks convincingly demonstrated that a deliberate seeding of selected impurities can have a positive effect on the plasma performance. On the one hand, a significant reduction of the head load on divertor plates, one of the main concerns by constructing a thermonuclear reactor, ${ }^{1}$ has been achieved due to the increase of edge radiation. On the other hand, in some devices impurity seeding has led to an improvement of the energy confinement and the so-called radiation improved mode has been established with the same or even better confinement than in the $H$-mode. ${ }^{2}$ However, in order to make use of these positive impacts, the behavior of seeded impurity has to be strictly controlled and negative developments such as the accumulation of impurity ions in the plasma core accompanied by a strong increase of the central radiation losses should be avoided.

Plasma heating by radio-frequency (rf) waves has been proven to be a useful tool to control the behavior of puffed impurities. As it was demonstrated on the tokamak JET, this technique can effectively prevent accumulation, affecting impurity ions in different respects, in particular, by modifying the mechanisms of the particle and the heat transport. ${ }^{3,4}$ The most striking effect of rf waves on impurities has been observed in the tokamak TFR (Ref. 5) by creating a mode conversion layer on top of the second harmonic resonance of $\mathrm{Ar}^{16+}$. By operating in the mode conversion regime, it was possible to deposit the major part of the input power into seeded Ar impurity. As a result the majority of impurity par-

\footnotetext{
${ }^{a)}$ Permanent address: Department of Physics and Technology, Kharkiv National University, 61077 Kharkiv, Ukraine.
}

ticles gained a high enough energy and could leave the plasma along orbits, which deviate strongly in the radial direction due to inhomogeneities in the tokamak magnetic field. ${ }^{6}$ This led to a reduction of the impurity content in the plasma by several times.

In order to asses the prospective of impurity control by the rf waves in larger devices and under reactor conditions, proper modeling approaches have to be developed. One of the important parameters, which should be evaluated, is the averaged energy or temperature of heated impurity ions, $T_{z}$. The latter determines, in particular, the power transported to the main species, and, thus, the heating efficiency. Besides, $T_{z}$ characterizes the intensity of orbit losses for heated impurities.

Up to now, impurity temperature has been disregarded in transport modeling and is normally assumed to be equal to the temperature of the main ions, $T_{i}$. Under conditions of strong power absorption by impurities, this assumption can be violated. This could also be the case in fusion plasmas of a very low collisionality. ${ }^{7}$ In such a situation, $T_{z}$ should be determined by considering the heat balance for the impurity species in question. By doing this, one can confront difficulties originating in the behavior of the coefficient at highest (second-order) derivative of $T_{z}$ with respect to the minor radius $r$, which is proportional to the heat conduction $\kappa_{z}$. The heat conduction coefficient $\kappa_{z}$ varies normally stronger with the impurity ion species density $n_{z}$ than other terms in the heat transport equation. Therefore, the importance of this term and the actual order of the heat transport equation depend essentially on the impurity concentration. The density profiles for not fully stripped impurity species have a shelllike structure (see, e.g., Refs. 8 and 9 ) and $n_{z}$ decays by some 
orders of magnitude with the distance from the position where the ions in question are predominantly generated. Therefore the terms with $\kappa_{z}$ decrease much stronger than other and the actual order of the heat balance equation varies with the radial position.

In the present paper the situation described above is modeled by constructing a hierarchy of approximate solutions to the heat transport equation for the impurity species in question. A zero approximation for $T_{z}, T_{z}^{0}$, is obtained by solving the first-order differential equation without the heat conduction term. The boundary condition to this equation is prescribed by the properties of the convective contribution to the heat flux being proportional to the impurity particle flux $\mathrm{E}_{z}$. With $T_{z}^{0}$ known, one can find the next approximation $T_{z}^{1}$ by including into the first-order differential equation the terms with $\kappa_{z}$ where $T_{z}$ is replaced by $T_{z}^{0}$. As the next step, the regions, where the terms with $\kappa_{z}$ are of importance, are determined by comparing $T_{z}^{0}$ and $T_{z}^{1}$ and the corrected solution $T_{z}^{2}$ is obtained by solving in these regions the complete heat transport equation of the second order. The remainder of the paper is organized as follows. In the following section the particle and the heat transport equations for impurity ions are formulated. The elaborated procedure to calculate the impurity temperature is outlined in Sec. III. In Sec. IV the results of numerical calculations are presented and the important parametric dependencies are investigated. Conclusions are drawn in Sec. V.

\section{TRANSPORT EQUATIONS FOR IMPURITY IONS}

Consider a certain impurity species with the charge $z$ characterized by the density $n_{z}$ and the temperature $T_{z}$. The stationary variation of these parameters with the plasma minor radius $r$ is governed by the continuity and the heat balance equations:

$$
\begin{aligned}
\frac{1}{r} \frac{\partial}{\partial r}\left(r \Gamma_{z}\right)= & S_{z+1}+S_{z-1}-L_{z} \\
\frac{1}{r} \frac{\partial}{\partial r}\left(r q_{z}\right)= & Q_{z}+\frac{3}{2}\left[S_{z-1} T_{z-1}+S_{z+1} T_{z+1}-L_{z} T_{z}\right. \\
& \left.-n_{z} \sum_{\beta} \nu_{z \beta}^{\varepsilon}\left(T_{z}-T_{\beta}\right)\right],
\end{aligned}
$$

where

$$
\Gamma_{z}=-D_{z} \frac{\partial n_{z}}{\partial r}+V_{z} n_{z}
$$

is the density of the impurity particle flux with $D_{z}$ and $V_{z}$ being the diffusivity and the convection velocity, $S_{z-1}$ and $S_{z+1}$ are the contributions to the particle source due to ionization and recombination of the ions of the lower and the higher charges, respectively, and $L_{z}$ is the sink due to the same processes with the ion species in question:

$$
S_{z-1}=n c_{z-1}^{i} n_{z-1}, \quad S_{z+1}=n c_{z+1}^{r} n_{z+1}, \quad L_{z}=n\left(c_{z}^{i}+c_{z}^{r}\right) n_{z} .
$$

Here, $n$ is the electron density and $c_{z-1, z, z+1}^{i, r}$ are the rate coefficients of ionization and recombination, respectively. The density of the impurity heat flux is

$$
q_{z}=-\kappa_{z} \frac{\partial T_{z}}{\partial r}+\frac{3}{2} \Gamma_{z} T_{z},
$$

$\nu_{z \beta}^{\epsilon}$ is the frequency of the energy exchange with other plasma species $\beta$ by Coulomb collisions given in Eq. (20.6) of Ref. 10, and $Q_{z}$ is the heating power density due to ion cyclotron resonance with $\mathrm{rf}$ waves.

The boundary conditions to Eqs. (1) and (2) imply zero derivatives $\partial_{r} n_{z}$ and $\partial_{r} T_{z}$ at the plasma axis, $r=0$, and given $e$-folding lengths, $1 / \partial_{r}\left(\ln n_{z}\right)$ and $1 / \partial_{r}\left(\ln T_{z}\right)$, at the last closed magnetic surface or magnetic separatrix, $r=a$.

By combining the above equations, one gets the following, in the form convenient for further analysis:

$$
\begin{gathered}
S_{z-1} T_{z-1}+S_{z+1} T_{z+1}+T_{z}\left(S_{z-1}+S_{z+1}\right)-n_{z} \sum_{\beta} \nu_{z \beta}^{\varepsilon}\left(T_{z}-T_{\beta}\right) \\
+\frac{2}{3} Q_{z}=\left(\Gamma_{z}-\frac{2}{3} \frac{\kappa_{z}}{r}-\frac{2}{3} \frac{d \kappa_{z}}{d r}\right) \frac{d T_{z}}{d r}-\frac{2}{3} \kappa_{z} \frac{d^{2} T_{z}}{d r^{2}} .
\end{gathered}
$$

\section{COMPUTATION OF IMPURITY TEMPERATURE}

As is mentioned above, the variation of different terms in Eq. (3) with the impurity density is of importance for choosing an approach to solve this equation. The radial variation of $n_{z}$ is governed by Eq. (1), and we first analyze this qualitatively. Impurity particles enter the plasma as neutrals and are consequently ionized into higher charge states. Thus, by moving from the boundary, $r=a$, toward the plasma center, one should expect that $n_{z}$ increases at first and than decays. Depending on $z$ and the plasma parameters, the maximum and the minimum values of $n_{z}$ can differ by several orders of magnitude. The maximum $n_{z}$ is located normally at the position where the electron temperature $T_{e}$ is half of the ionization potential for the particles in question (see Refs. 11 and 12). For $T_{e}$ lower and higher, this value $n_{z}$ decays fast. This behavior is characteristic for species of not too high charges whose density does not approache a maximum at the plasma axis. Figure 1 illustrates this by displaying the density profiles of several ionization states and corresponding fluxes for argon impurity seeded into the JET plasmas. They were found by using the transport code RITM (Ref. 9) for the plasma conditions described in Sec. IV.

The terms on the left-hand side (lhs) of Eq. (3) are independent or vary linearly with $n_{z}$. Consider the transport contributions collected on the right-hand side (rhs). Normally, one distinguishes neoclassical and anomalous components of these, due to collisions and turbulence, respectively. ${ }^{11}$ Concerning the first convective term, one has to take into account that the neoclassical contributions to the particle diffusion and convection velocity, $D_{z}$ and $V_{z}$, include terms due to interactions with the background hydrogen ions ${ }^{7}$ nearly independent of $n_{z}$ when $n_{z} \ll n_{i}$. Therefore, $\Gamma_{z}$ contains, independently of anomalous contributions included, terms varying linearly with $n_{z}$ and its derivative.

On the contrary, the neoclassical heat conduction is due to mutual collisions of particles of a particular plasma component, ${ }^{13}$ i.e., $\kappa_{z}^{\text {neo }} \sim n_{z} \nu_{z z}^{\varepsilon}$. An exception is the so-called "plateau" regime where the transport coefficients are formally independent of the collision frequency and one expects 

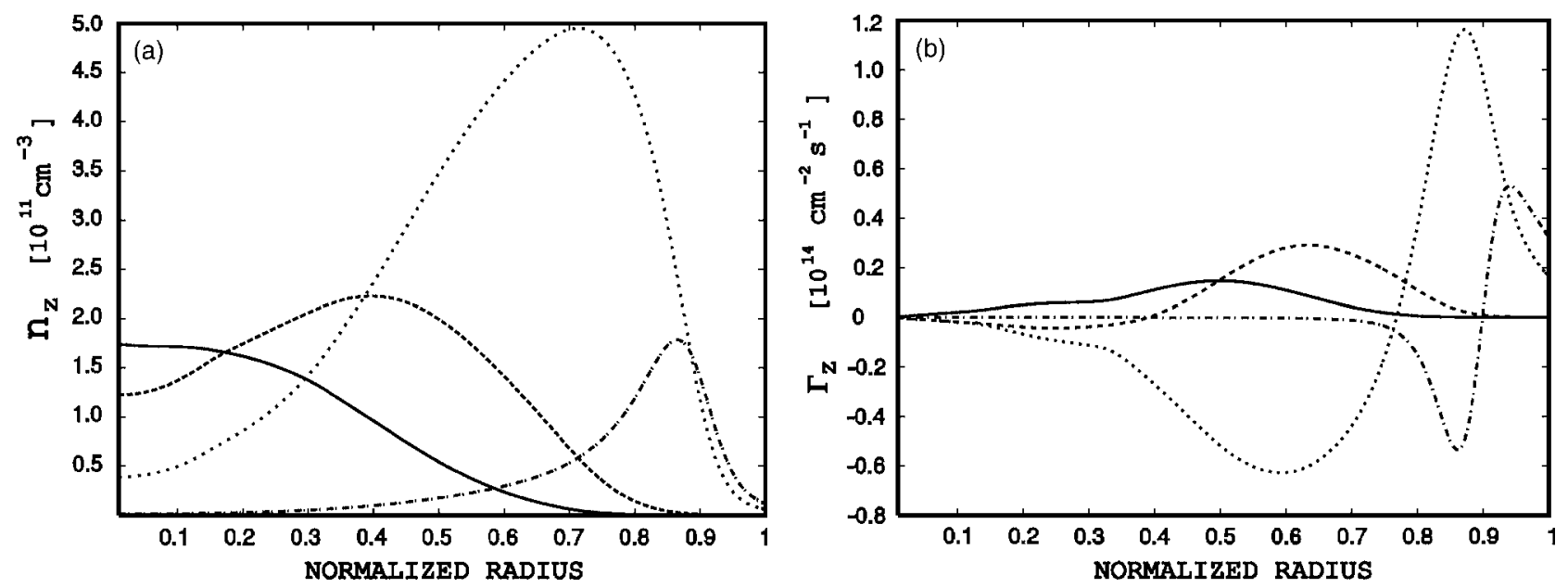

FIG. 1. Radial profiles of the particle (a) and the flux (b) densities for $\operatorname{Ar}^{18+}$ (solid line), $\operatorname{Ar}^{17+}$ (dashed line), $\operatorname{Ar}^{16+}$ (dot line), and $\mathrm{Ar}^{15+}$ (dash-dot line) charged states.

$\kappa_{z}^{\text {neo }} \sim n_{z}$. Nevertheless, this is not the case since impurities of the charged state in question collide also with the main ions. Therefore the usual neoclassical heat conductivity computed under the assumption that only the $z$ ions are presented in the plasma ${ }^{13}$ should be corrected by the factor $\nu_{z z}^{\varepsilon} /\left(\nu_{z z}^{\varepsilon}\right.$ $\left.+\nu_{z i}^{\varepsilon}\right)$. This is roughly proportional to $n_{z}$ when $\nu_{z z} \leqslant \nu_{z i}$ and $\kappa_{z}^{\text {neo }} \sim\left(n_{z}\right)^{\gamma_{\text {neo }} \text { with }} \gamma_{\text {neo }} \geqslant 2$.

An anomalous heat conduction of impurity ions, $\kappa_{z}^{\text {an }}$, is a completely unexplored subject both from theoretical and experimental points of view. Nevertheless, one can expect such a contribution, in particular, from the impurity branch of ion temperature gradient (ITG) drift instability. ${ }^{14,15}$ The growth rate of this branch and, thus, the corresponding heat diffusivity component $\chi_{\text {an }}$ are proportional to the impurity concentration. Therefore, $\kappa_{z}^{\text {an }}=n_{z} \chi_{\text {an }} \sim\left(n_{z}\right)^{\gamma_{\text {an }}}$ with $\gamma_{\text {an }} \geqslant 2$.

Thus, one should generally expect that the heat conductivity of an impurity species, whose density changes significantly through the plasma, varies much stronger than other terms in Eq. (3) for the impurity temperature. This would mean that the coefficient at the highest derivative in Eq. (3) can become so small that the actual order of this differential equation changes. If this is not taken into account in the integration procedure, numerical problems arise.

In order to overcome these difficulties, we construct a hierarchy of approximate solutions to Eq. (3). First, we look for the solution $T_{z}^{0}(r)$ to Eq. (3) without the heat conduction terms on the rhs:

$$
\begin{aligned}
& S_{z-1} T_{z-1}+S_{z+1} T_{z+1}+T_{z}^{0}\left(S_{z-1}+S_{z+1}\right)-n_{z} \sum_{\beta} \nu_{z \beta}^{\varepsilon}\left(T_{z}^{0}-T_{\beta}\right) \\
& \quad+\frac{2}{3} Q_{z}=\Gamma_{z} \frac{d T_{z}^{0}}{d r} .
\end{aligned}
$$

In order to integrate this equation of the first order, one requires a boundary condition. However, this cannot be one of the conditions to Eq. (3). On the one hand, any solution to Eq. (4) automatically satisfies the requirement of a zero derivative at $r=0$. Indeed, differentiating Eq. (4) once more and taking into account that $\Gamma_{z}$ and derivatives of all other parameters independent of $T_{z}^{0}$ reduce to zero at the plasma axis, lead to $\left(T_{z}^{0}\right)_{r}^{\prime}=0$ at $r=0$. On the other hand, most probably, $T_{z}^{0}$ cannot satisfy the boundary condition at the separatrix, the prescribed $e$-folding length. Indeed, one can see in Fig. 1(b) that in addition to $r=0, \Gamma_{z}$ reduces to zero at some another point $r_{z}$ in the vicinity of the maximum $n_{z}$. By integrating Eq. (4) from the separatrix, $r=a$, with a prescribed $e$-folding length one can come, only by chance, to a zero lhs at $r=r_{z}$ required by zero rhs here. At the same time, the requirement of zero lhs at $r=r_{z}$ itself provides a necessary boundary or, more exactly, intermediate condition to Eq. (4):

$$
T_{z}^{0}\left(r_{z}\right)=\frac{2 / 3 Q_{z}+\sum_{\beta} \nu_{z \beta}^{\varepsilon} n_{z} T_{\beta}+S_{z-1} T_{z-1}+S_{z+1} T_{z+1}}{\sum_{\beta} \nu_{z \beta}^{\varepsilon} n_{z}+S_{z-1}+S_{z+1}} .
$$

By integrating Eq. (4) into both directions from $r=r_{z}$ one gets the whole profile of $T_{z}^{0}(r)$.

The next approximation to $T_{z}, T_{z}^{1}$, is determined by solving, with the procedure described above, Eq. (3) where the terms with the heat conduction are computed using $T_{z}^{0}$, i.e.,

$$
\begin{aligned}
S_{z-1} T_{z-1} & +S_{z+1} T_{z+1}+T_{z}^{1}\left(S_{z-1}+S_{z+1}\right)-n_{z} \sum_{\beta} \nu_{z \beta}^{\varepsilon}\left(T_{z}^{1}-T_{\beta}\right) \\
& +\frac{2}{3} Q_{z}+\delta=\Gamma_{z} \frac{d T_{z}^{1}}{d r}
\end{aligned}
$$

where

$$
\delta=\kappa_{z} \frac{d^{2} T_{z}^{0}}{d r^{2}}+\left(\frac{\kappa_{z}}{r}+\frac{d \kappa_{z}}{d r}\right) \frac{d T_{z}^{0}}{d r} .
$$

By computing the error $\epsilon(r)=\left|1-T_{z}^{1} / T_{z}^{0}\right|$, one can find the regions where it exceeds the prescribed tolerance level $\epsilon_{0}$. In such regions the total heat balance equation (2) has to be solved. Normally this can be done without numerical problems since the magnitude of $\kappa_{z}$ is large enough here. In order to find the final solution in each of such regions, the values of $T_{z}^{2}$ at the edges of the region are assumed equal to the values of $T_{z}^{1}$ there. 


\section{RESULTS OF CALCULATION AND DISCUSSION}

As an illustration, we apply the approach outlined above to compute the temperatures of argon impurity seeded into JET plasmas with the major and the minor radii of the separatrix, $R_{0}=2.96 \mathrm{~m}$ and $a=0.9 \mathrm{~m}$, respectively, the plasma current $I_{p}=2.5 \mathrm{MA}$, and the magnetic field $B_{T}=3.47 \mathrm{~T}$ at $R_{0}$. The plasma has been auxiliary heated by neutral beam injection with a power of $7.5 \mathrm{MW}$ and by radio waves with a frequency of $f=23.1 \mathrm{MHz}$ and a power of $2.5 \mathrm{MW}$. The latter should provide first harmonic resonances for several argon ion species at the following radial positions: $R=2.68 \mathrm{~m}$ for $\mathrm{Ar}^{16+}, R=2.85 \mathrm{~m}$ for $\mathrm{Ar}^{17+}$, and $R=3.02 \mathrm{~m}$ for $\mathrm{Ar}^{18+}$ ions. The deuterium fundamental cyclotron resonance was located at $R=3.36 \mathrm{~m}$. The radial profiles of the power density absorbed by impurity species have been computed with the TORIC code. ${ }^{16}$

The radial profiles of plasma parameters were calculated by the RITM code. ${ }^{9}$ In this code, the densities and the fluxes of the main and the impurity neutral particles, which enter the plasma volume through the separatrix, are computed by solving kinetic equations in a diffusion approximation. The densities of electrons $n_{e}$ and impurity ions $n_{z}$ are determined from corresponding continuity equations by taking into account the particle sources due to the ionization of neutrals. The radial distribution of the particle source due to neutral beam injection is taken from the experimental data. ${ }^{17}$ Presently the RITM code allows to consider all charged states of $\mathrm{He}, \mathrm{C}, \mathrm{O}, \mathrm{Ne}, \mathrm{Si}$, and $\mathrm{Ar}$ impurities. The density of the main ions, $n_{i}$, is found from the quasineutrality condition. The electron and the main ion temperatures, $T_{e}$ and $T_{i}$, are determined from the heat transport equations. The transport coefficients in RITM are determined by taking into account both the neoclassical and the anomalous contributions. The latter is evaluated by considering in a quasilinear approximation the most important unstable drift modes such as ion temperature gradient, trapped electron, and drift Alfvén and drift resistive ballooning microinstabilities. Several mechanisms for the suppression of these instabilities, e.g., by the radial electric field shear, density gradient, diamagnetic flows, are taken into account. This permits to describe both $L$ - and $H$-mode conditions including the edge transport barrier.

Figure 2 presents the radial profiles of the computed deuteron temperature and of the $\mathrm{Ar}^{17+}$ temperature determined in different approximations by the procedure described in the preceding section. One can see that there are, indeed, regions where the impurity heat conduction is of importance and has to be included into calculations, and regions where its role is small and has to be neglected. The approach developed in the present paper provides a possibility to take simultaneously both types of behavior into consideration. Figure 3 displays the radial profiles of the temperatures for the main ions and of $\mathrm{Ar}^{16+}$ and $\mathrm{Ar}^{18+}$ ion species.

The developed approach to the computation of impurity ion temperatures has been incorporated into the transport code RITM. An important unique feature of this combined model is the possibility to take into account the energy transfer from heated impurity ions to the main ones as well as to

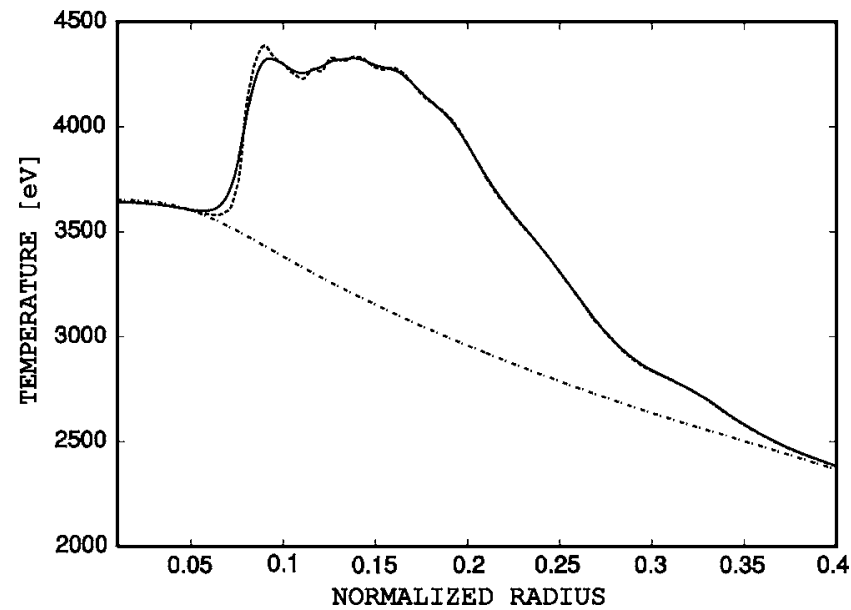

FIG. 2. The temperature of the main ions (dash-dot line) and of $\mathrm{Ar}^{17+}$ ions computed from the first-order (dashed line) and second-order (solid line) heat transport equations.

other impurity ions and accompanying modifications in the particle and energy transport. Finally, by coupling with the full wave code TORIC, which computes the profiles of the power density absorbed by different ions species, a selfconsistent description of the ICRH (ion cyclotron resonance heating) scenario is elaborated. Performed computations revealed, in particular, that heating of the main ions through collisions with the impurity ones results in an increase of anomalous transport due to ITG modes, as it is demonstrated in Fig. 4 by the radial profiles of the ion heat diffusivity at the beginning of the heating stage and in the steady state. As a result, the electron temperature is reduced and the population of different impurity charged states is modified, namely, the densities of $\mathrm{Ar}^{18+}$ and $\mathrm{Ar}^{17+}$ species are reduced and the $\mathrm{Ar}^{16+}$ density is increased. As a consequence, the absorption power profiles computed by TORIC code are also changed.

This is demonstrated in Figs. 5 and 6 by the profiles of the rf power density coupled with different impurity species computed at the beginning of ICRH and after the relaxation to the steady state. One can see a significant modification both of profiles and of the total power input.

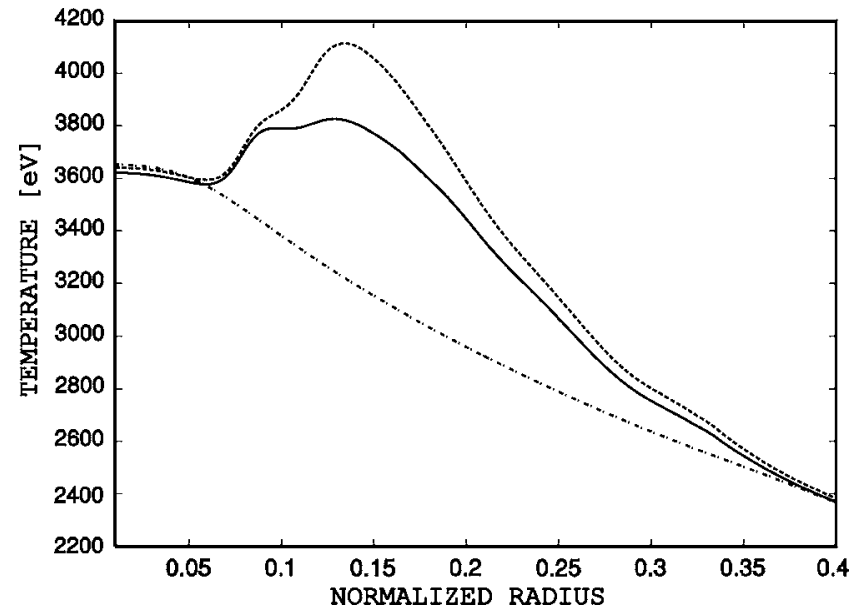

FIG. 3. The temperature of the main ions (dash-dot line), $\mathrm{Ar}^{16+}$ (solid line), and $\mathrm{Ar}^{18+}$ (dashed line) charged states. 


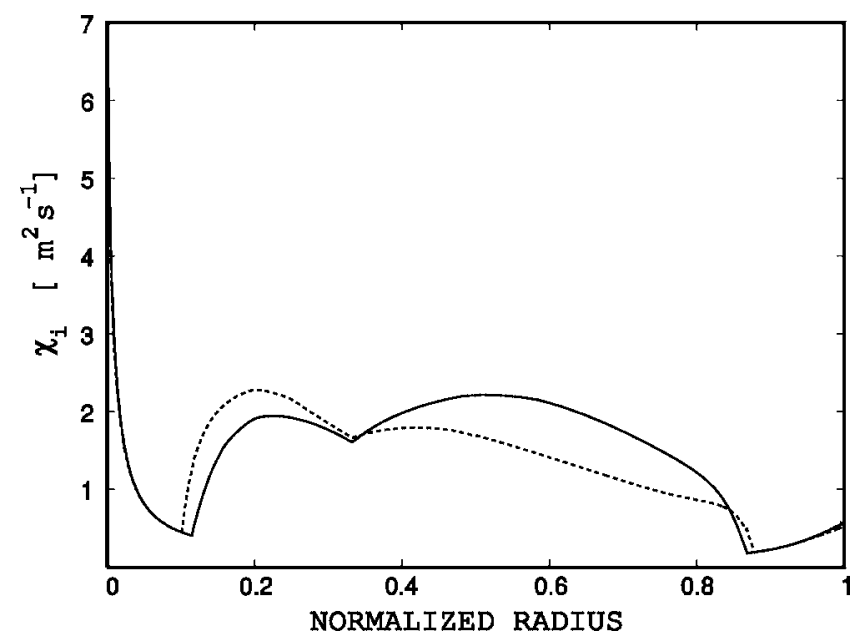

FIG. 4. The ion thermal diffusivity at the beginning of the ICRH phase (solid line) and in the steady state (dashed line).

By going to reactor relevant plasma conditions of a significantly lower collisionality, the coupling between the main and the impurity ion temperatures becomes weaker. Therefore, a stronger increase in $T_{z}$ can be achieved with the same level of impurity heating. This is demonstrated in Fig. 7, where we display the ratio of the ion temperatures, $T_{z} / T_{i}$, computed for $\mathrm{Ar}^{17+}$ species at the position of corresponding ion cyclotron resonance, under the assumption that $T_{i}$ is increased by a constant factor $\Theta$ with respect to the profile shown in Fig. 3.

Our results predict a significant difference, up to $1 \mathrm{keV}$ in JET, between the temperatures of the main and the impurity ions when the latter are selectively heated by the rf waves. A comparison of these predictions with the experimental data would provide an important check of the model. Unfortunately, such a comparison cannot be done presently. First, namely, the impurity ion temperature measured by the charge-exchange (CX) of impurity with neutral beams is normally taken as an estimate for the main ion temperature and

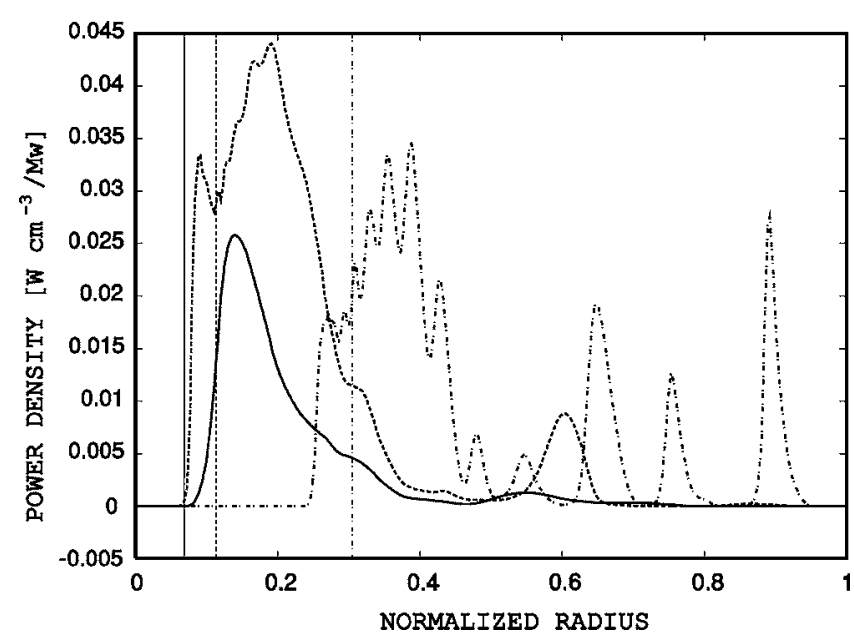

FIG. 5. The power density absorbed by $\mathrm{Ar}^{16+}$ (dash-dot line), $\mathrm{Ar}^{17+}$ (dashed line), and $\mathrm{Ar}^{18+}$ (solid line) ion species at the beginning of the ICRH phase. Vertical lines indicate the radial positions of the corresponding cyclotron resonances.

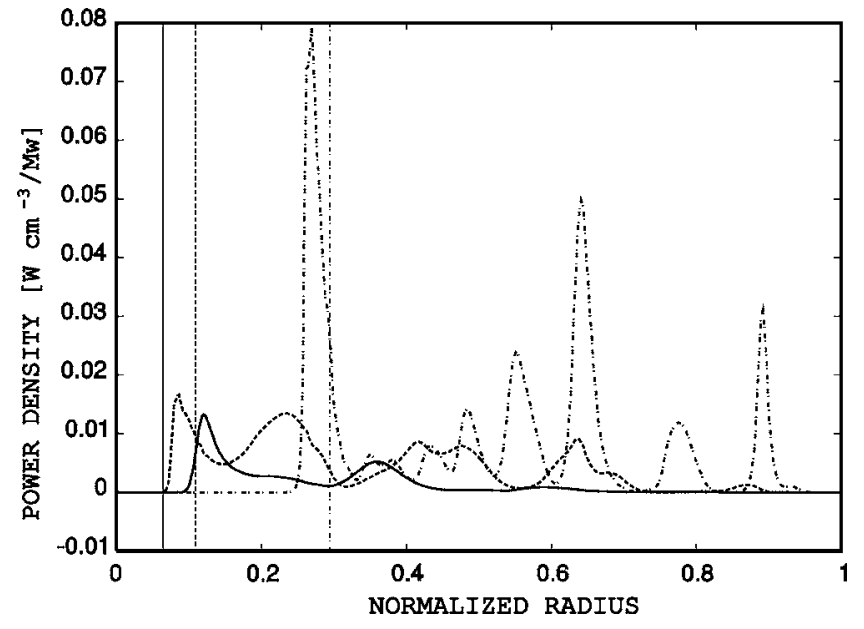

FIG. 6. The power density absorbed by $\mathrm{Ar}^{16+}$ (dash-dot line), $\mathrm{Ar}^{17+}$ (dashed line), and $\mathrm{Ar}^{18+}$ (solid line) ion species in the steady state. Vertical lines indicate the positions of the corresponding cyclotron resonances.

normally there are no other reliable approaches to measure $T_{i}$ independently. Second, in very few discharges performed on JET with a selective impurity heating, the CX diagnostic did not work properly. Our computations show, however, that reliable and independent methods to measure the temperatures of different ion species are of very importance for the conditions of strong impurity heating and have to be developed in future.

\section{CONCLUSION}

The developed approach allows us to calculate the temperature of impurity ions which is of particular interest under conditions when selective impurity charged states are strongly heated by the rf waves. It is demonstrated that, on the one hand, in the vicinity of cyclotron resonances, where the power is predominantly absorbed, a strong radial gradient of the impurity temperature can arise and the impurity heat conduction can play an important role in the energy trans-

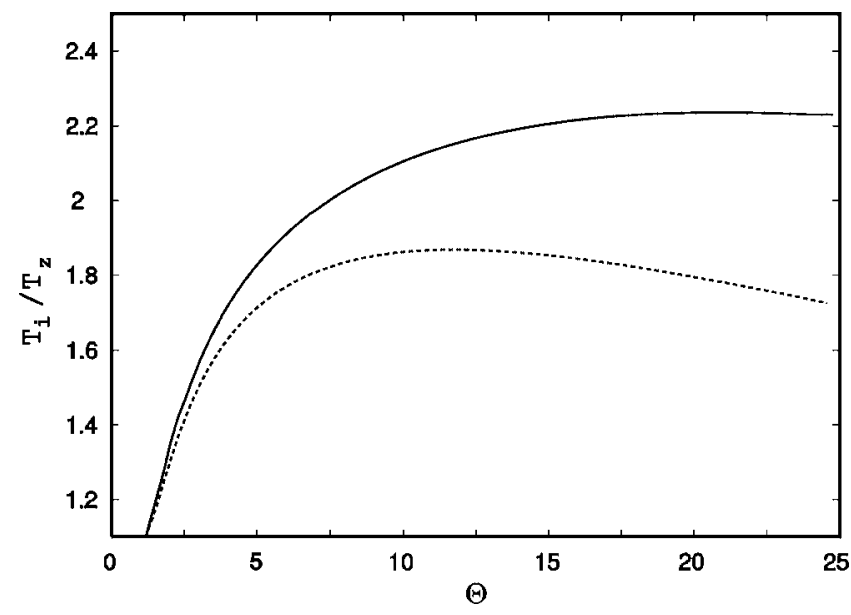

FIG. 7. The ratio $T_{z} / T_{i}$ computed for $\mathrm{Ar}^{17+}$ ion species at the position of cyclotron resonance $R_{\mathrm{Ar}}{ }^{17+}$, with the main ion temperature increased by the factor $\Theta$ with respect to the profile in Fig. 3. Solid curves are obtained by computing $T_{z}$ from the first-order impurity heat transport equation and dashed curves are from the second-order one. 
port. On the other hand, beyond such regions the contribution from the impurity heat conduction is negligibly small. The corresponding variation with the minor radius of the actual order of the impurity heat transport equation is taken into account in the approach elaborated. This method has been incorporated into the transport code RITM and computations for JET discharges with impurity seeding have been performed. A self-consistent description of the ICRH scenario for minority argon impurity is established by computing the attenuated power in the full wave code TORIC with the plasma parameter profiles calculated by the RITM code. It is shown that such a self-consistency is of much importance since the modification in the particle and heat transport caused by the heating results in significant changes of the absorbed power profile.

\section{ACKNOWLEDGMENT}

The authors thank Dr. M. Brambilla from IPP Garching for the opportunity of using the TORIC code.
${ }^{1}$ G. Federici, C. H. Skinner, J. N. Brooks et al., Nucl. Fusion 41, 1967 (2001).

${ }^{2}$ J. Ongena, R. Budny, P. Dumortier et al., Phys. Plasmas 8, 2188 (2001).

${ }^{3}$ M. F. F. Nave, J. Rapp, T. Bolzonella et al., Nucl. Fusion 43, 1204 (2003).

${ }^{4}$ M. E. Puiatti, M. Mattioli, G. Telesca et al., Plasma Phys. Controlled Fusion 44, 1863 (2002).

${ }^{5}$ TFR Group, Nucl. Fusion 22, 956 (1982).

${ }^{6}$ D. S. Darrow, R. Majeski, N. J. Fisch, R. F. Heeter, H. W. Herrmann, M. C. Herrmann, M. C. Zarnstorff, and S. J. Zweben, Nucl. Fusion 36, 509 (1996).

${ }^{7}$ K. W. Wenzel and D. J. Sigmar, Nucl. Fusion 30, 1117 (1990).

${ }^{8}$ T. Tazima, Y. Nakamura, and K. Inoue, Nucl. Fusion 17, 419 (1977).

${ }^{9}$ M. Z. Tokar, Plasma Phys. Controlled Fusion 36, 1819 (1994).

${ }^{10}$ B. A. Trubnikov, Reviews of Plasma Physics (Consultants Bureau, New York, 2004), Vol. 1.

${ }^{11}$ J. Wesson, Tokamaks, 2nd ed. (Clarendon, Oxford, 1997).

${ }^{12}$ M. Z. Tokar, Nucl. Fusion 34, 853 (1994).

${ }^{13}$ C. S. Chang and F. L. Hinton, Phys. Fluids 29, 3314 (1986).

${ }^{14}$ R. R. Dominguez, Nucl. Fusion 31, 2063 (1991).

${ }^{15}$ M. R. Wade, W. A. Houlberg, and L. R. Baylor, Phys. Rev. Lett. 84, 282 (2000).

${ }^{16}$ M. Brambilla, Nucl. Fusion 34, 1121 (1994).

${ }^{17}$ B. Unterberg, D. Kalupin, M. Z. Tokar et al., Plasma Phys. Controlled Fusion 46, A241 (2004). 